\title{
Uterine Sarcomas
}

\author{
Rita Lucas and Teresa Margarida Cunha
}

\section{Contents}

1 Epidemiology

2 Pathology

2.1 Smooth Muscle Tumours

2.2 Endometrial Stromal Tumours....

2.3 Mixed Epithelial and Mesenchymal

Tumours

2.4 Miscellaneous Mesenchymal Tumours.....

3 Clinical Background

4 Staging.

5 Imaging

5.1 Leiomyosarcoma

5.2 Endometrial Stromal Sarcomas

5.3 Adenosarcoma.

6 Prognosis and Treatment

References

\section{$1 \quad$ Epidemiology}

000

000

000

000

000

000

000

000

000

000

000

000

000

000
R. Lucas $(\bowtie)$

Hospital dos Lusíadas de Lisboa, Lisbon, Portugal e-mail: ritalucas1@gmail.com

\section{T.M. Cunha}

Instituto Português de Oncologia de Lisboa

Francisco Gentil, Lisbon, Portugal

e-mail: tmargarida@gmail.com
The term sarcoma comes from a Greek word meaning "fleshy growth".

Gynaecological sarcomas are uncommon tumours with aggressive behaviour that account for approximately $1 \%$ of all female genital tract malignancies and 3-7\% of uterine cancers (D'Angelo and Prat 2010; Major et al. 1993). Although rare, the most frequent of all gynaecological sarcomas is leiomyosarcoma.

The great majority of sarcomas arise in the uterus and are classified according to the histologic tissue from which they are derived, either smooth muscle or endometrial stroma. Although uncommon, the histopathological diversity of gynaecological sarcomas have contributed to many inaccuracies and lack of consensus in nomenclature in the past.

Sarcomas can also develop at other sites in the female genital tract with different features, for example, ovarian fibrosarcoma, vulvovaginal leiomyosarcoma or rhabdomyosarcoma; however these tumours are extremely rare and are outside the scope of this chapter.

\section{Pathology}

The current World Health Organization (WHO) classification of tumours of the female reproductive organs (Kurman et al. 2014), recently revised and published in 2014, classifies mesenchymal 
tumours of the uterine corpus into smooth muscle origin, endometrial stromal origin, mixed epithelialmesenchymal origin and miscellaneous tumours.

In comparison with the previous WHO 2003 classification, the new classification reintroduces high-grade endometrial stromal sarcoma as a separate entity, supported by emerging recent molecular and morphological data, and replaces the designation "undifferentiated endometrial sarcoma" by undifferentiated uterine sarcoma to reflect the fact that an endometrial origin is not certain (McCluggage et al. 2014).

Uterine sarcomas more frequently exhibit early peritoneal and haematogenous spread with distant metastases, instead of starting to spread locally in the pelvis and to lymph nodes as carcinomas do (Seddon and Davda 2011).

\subsection{Smooth Muscle Tumours}

Smooth muscle tumours are composed of cells showing smooth muscle differentiation arising within the myometrium that can be either benign or malignant. Because they arise in the stroma, the classic histological criteria for invasion are not so easy to define as in epithelial tumours.

This designation comprises:

- Classic benign leiomyoma (i.e., the most frequent uterine tumour)

- Smooth muscle tumour of uncertain malignant potential (STUMP), also called atypical smooth muscle neoplasm, a lesion not fulfilling entirely the criteria for a leiomyoma or leiomyosarcoma

- Leiomyosarcoma, a malignant tumour that occasionally shows epithelioid or myxoid features

Leiomyosarcomas and leiomyomas are independent entities with differing cytogenetic abnormalities that can however coexist (McCluggage et al. 2014; Bodner et al. 2003). Classical teaching books state that malignant transformation should be suspected in rapid growth of a leiomyoma in a menopausal woman; however the most recent evidence supports that uterine sarcomas do not generally arise from pre-existing leiomyomas, with only very rare exceptions (about $0.2 \%$ of cases). By consensus from genetic studies, the current concept is that most sarcomas arise independently (Hodge and Morton 2007; McCluggage 2002a). Furthermore, rapid growth can also occur in premenopausal women in leiomyoma.

\subsection{Endometrial Stromal Tumours}

Endometrial stromal neoplasms are a subset of uterine mesenchymal neoplasms that account for less than $10 \%$ of all uterine sarcomas (Chan et al. 2008). These rare uterine neoplasms mimic proliferative endometrium; however, they have myometrial and/or vascular invasion (Kurman et al. 2014).

Recent molecular genetics and immunohistochemistry advances have improved the understanding of these lesions and helped to redefine the WHO classification into more meaningful categories with distinct prognosis (Kurman et al. 2014; Ali and Rouzbahman 2015; Conklin and Longacre 2014; Xue and Cheung 2011), namely

- Low-grade endometrial stromal sarcoma

- High-grade endometrial stromal sarcoma

- Undifferentiated uterine sarcoma

In tumours in which a focal smooth muscle differentiation coexists, the neoplasm is considered an endometrial stromal sarcoma if the smooth muscle component involves $<30 \%$ of the total lesion volume. Tumours composed of a larger smooth muscle component are designated as "mixed endometrial stromal and smooth muscle neoplasms" or stromomyomas. However, there is still few data regarding these lesions (Kurman et al. 2014).

\subsection{Mixed Epithelial and Mesenchymal Tumours}

This category includes adenosarcoma, a rare tumour that accounts for 5-9\% of uterine sarcomas. In this lesion the epithelial component is benign or atypical, and the stromal component 
is low-grade malignant (Tse et al. 2011). It is a difficult diagnosis to make requiring an experienced gynaecological pathologist (Blom and Guerrieri 1999).

Even though adenosarcomas typically have a low malignant potential, there is however a subgroup defined as having sarcomatous overgrowth, with pure sarcoma occupying at least $25 \%$ of the tumour (McCluggage et al. 2014).

Carcinosarcoma, previously called malignant Müllerian tumour, has recently been reclassified as a dedifferentiated or metaplastic form of endometrial carcinoma (type II cancer). In addition, the epidemiology, risk factors, and clinical behaviour associated with carcinosarcoma suggest a closer relationship to endometrial carcinoma than to other sarcomas (McCluggage 2002a, b).

\subsection{Miscellaneous Mesenchymal Tumours}

This group includes rhabdomyosarcoma, which is a notably rare tumour that shows skeletal muscle differentiation, occurring both in the vagina and uterine corpus and cervix, typically arising in children and adolescents.

For this chapter only, malignant uterine sarcomas with mesenchymal and mixed epithelialmesenchymal origin will be considered as summarized in Table 1.

Table 1 Malignant uterine sarcomas

\begin{tabular}{l|l}
\hline Mesenchymal origin & $\begin{array}{l}\text { Mixed epithelial and } \\
\text { mesenchymal origin }\end{array}$ \\
\hline Smooth muscle origin & Adenosarcoma \\
\hline Leiomyosarcoma & \\
\hline $\begin{array}{l}\text { Endometrial stroma and } \\
\text { related origin }\end{array}$ & \\
\hline $\begin{array}{l}\text { Low-grade endometrial } \\
\text { stromal sarcoma }\end{array}$ & \\
\hline $\begin{array}{l}\text { High-grade endometrial } \\
\text { stromal sarcoma }\end{array}$ & \\
\hline $\begin{array}{l}\text { Undifferentiated uterine } \\
\text { sarcoma }\end{array}$ & \\
\hline
\end{tabular}

\section{$3 \quad$ Clinical Background}

The clinical presentation of uterine sarcomas is non-specific, with a peak incidence between 50 and 65 years of age. Of note, low-grade endometrial stromal sarcomas occur mainly in premenopausal women (D'Angelo and Prat 2010; Conklin and Longacre 2014).

Frequently there is history of a rapidly growing uterine mass, abnormal vaginal bleeding and pelvic or abdominal pain.

Less frequently, leiomyosarcoma can present hemoperitoneum (due to tumour rupture), or symptoms resulting from extrauterine extension or metastases (D'Angelo and Prat 2010).

The diagnosis of uterine sarcomas is frequently unexpected, discovered incidentally on histopathology analysis following hysterectomy, often for benign diseases. Of those patients undergoing hysterectomy for a preoperative diagnosis of leiomyoma, $0.5 \%$ is subsequently diagnosed with leiomyosarcoma (Wu et al. 2011).

African-american women have an approximately twofold higher incidence of leiomyosarcomas (but not other types of uterine sarcoma) than caucasian women (Sherman and Devesa 2003).

Occasional cases are associated with long-term tamoxifen therapy, polycystic ovarian disease, prolonged estrogenic stimulation or pelvic irradiation. There are also some associated hereditary conditions such as hereditary leiomyomatosis/renal cell carcinoma syndrome and survivors of childhood retinoblastoma (Toro et al. 2003; Yu et al. 2009).

Obesity, diabetes and younger age at menarche have been associated with increased risk of endometrial stromal sarcomas, although the molecular mechanisms involved are yet to be elucidated (Felix et al. 2013).

Compared with the more common endometrial carcinomas (epithelial neoplasms), uterine sarcomas behave aggressively and are associated with a poorer prognosis.

\section{$4 \quad$ Staging}

Staging is surgical, including total hysterectomy with bilateral salpingo-oophorectomy, and remains the most powerful prognostic factor for 
Table 22009 FIGO staging systems for uterine sarcomas

\begin{tabular}{|c|c|}
\hline \multicolumn{2}{|c|}{ Uterine leiomyosarcoma and endometrial stromal sarcoma } \\
\hline Stage I & Tumour limited to uterus \\
\hline IA & $\leq 5 \mathrm{~cm}$ \\
\hline IB & $>5 \mathrm{~cm}$ \\
\hline Stage II & Tumour extends beyond the uterus, within the pelvis \\
\hline IIA & Adnexal involvement \\
\hline IIB & Involvement of other pelvic tissues \\
\hline Stage III & Tumour invades abdominal tissues (not just protruding into the abdomen) \\
\hline IIIA & One site \\
\hline IIIB & More than one site \\
\hline IIIC & Metastasis to pelvic and/or para-aortic lymph nodes \\
\hline \multicolumn{2}{|l|}{ Stage IV } \\
\hline IVA & Tumour invades bladder and/or rectum \\
\hline IVB & Distant metastasis \\
\hline \multicolumn{2}{|c|}{ Uterine adenosarcoma } \\
\hline Stage I & Tumour limited to uterus \\
\hline IA & Tumour limited to endometrium/endocervix with no myometrial invasion \\
\hline IB & Less than or equal to half myometrial invasion \\
\hline IC & More than half myometrial invasion \\
\hline Stage II & Tumour extends beyond the uterus, within the pelvis \\
\hline IIA & Adnexal involvement \\
\hline IIB & Involvement of other pelvic tissues \\
\hline Stage III & Tumour invades abdominal tissues (not just protruding into the abdomen) \\
\hline IIIA & One site \\
\hline IIIB & More than one site \\
\hline IIIC & Metastasis to pelvic and/or para-aortic lymph nodes \\
\hline \multicolumn{2}{|l|}{ Stage IV } \\
\hline IVA & Tumour invades bladder and/or rectum \\
\hline IVB & Distant metastasis \\
\hline
\end{tabular}

uterine sarcomas. However in pretreatment evaluation, staging largely depends on imaging as the clinical evaluation is very limited.

Due to their different biologic behaviour, in 2009 FIGO introduced two new dedicated staging systems, specifically designed for uterine sarcomas, in which the morphological tumour subtype determines which staging system is used: one staging system for leiomyosarcoma and endometrial stromal sarcoma and a different system for adenosarcoma (Prat 2009a, b) (Table 2).

Adenosarcoma tends to arise at the endometrial or cervical surface, progressively invading the myometrium or cervical stroma in a similar way to endometrial carcinomas. The staging system is comparable to endometrial carcinomas, whereas leiomyosarcoma and endometrial stromal sarcoma that usually arise within the myometrium and have a different tumoural progression are staged in a different fashion.

\section{$5 \quad$ Imaging}

Imaging plays an important role in the evaluation of disease extent and treatment decision, even though the diagnosis relies on the histological examination. Endometrial sampling may yield the correct diagnosis but not in all patients: cases that arise within the endometrium may be indistinguishable from endometrial carcinoma, whereas those that arise from the myometrium may be indistinguishable from degenerating leiomyomas (Sala et al. 2013). 
Table 3 Key points in the evaluation of sarcomatous uterine lesions

\begin{tabular}{l|l}
\hline Tumour limits/interface & $\begin{array}{l}\text { Early-stage uterine leiomyosarcomas can be well-circumscribed, while more advanced } \\
\text { lesions typically have an irregular contour. On the other hand, endometrial stromal } \\
\text { sarcomas may exhibit a diffusely infiltrative pattern of myometrial invasion }\end{array}$ \\
\hline $\begin{array}{l}\text { Depth of myometrial } \\
\text { invasion }\end{array}$ & $\begin{array}{l}\text { Important in the substaging of stage I adenosarcomas: Stage IA tumours are limited to } \\
\text { the endometrium, stage IB invades less than or half of myometrium thickness and stage } \\
\text { IC equates to more than one half myometrial invasion. It is also considered a risk factor } \\
\text { for recurrence } \\
\text { Since most other uterine sarcomas are predominantly myometrial-based lesions, } \\
\text { myometrial invasion is not considered in the staging of these neoplasms }\end{array}$ \\
\hline Cervical involvement & It has an adverse influence on prognosis, for example, in the case of a leiomyosarcomas \\
\hline Adnexal involvement & $\begin{array}{l}\text { Adnexal involvement affects the tumour stage (FIGO stage IIA) and may occur as } \\
\text { result of direct extension or metastatic spread of tumour }\end{array}$ \\
\hline Lymph nodes & Pelvic or para-aortic lymph node involvement upstages uterine sarcomas to stage IIIC \\
\hline $\begin{array}{l}\text { Involvement of pelvic } \\
\text { tissues }\end{array}$ & $\begin{array}{l}\text { Other sites of pelvic tumour involvement should be documented since this equates to } \\
\text { FIGO stage IIB }\end{array}$ \\
\hline $\begin{array}{l}\text { Involvement of omentum } \\
\text { and other abdominal } \\
\text { tissues }\end{array}$ & $\begin{array}{l}\text { The number of affected locations in peritoneal tissue is important as FIGO stage IIIA } \\
\text { equates to one site of abdominal involvement and IIIB to more than one site }\end{array}$ \\
\hline
\end{tabular}

Pre-surgical subtyping of uterine sarcomas is very important for therapeutic management. Even though the imaging findings of uterine sarcomas have a considerable overlap, there are however some specific features that can narrow the differential diagnosis list.

Ultrasound is usually the first-line study to evaluate women for potential uterine pathology as it is a readily available, non-invasive and relatively inexpensive technique. It gives an initial evaluation of the size and location of the lesion and may identify features suggestive of aggressiveness; however, the accuracy is very low.

Computed tomography (CT) is mainly used for staging in the assessment of extra-pelvic disease, distant metastases and follow-up.

The primary modality for imaging uterine sarcomas is magnetic resonance imaging (MRI) due to the improved ability of this technique to delineate local disease.

When evaluating a possible sarcomatous uterine lesion with MRI, there are some specific key points that are important to evaluate as described in Table 3 (McCluggage et al. 2014).

\subsection{Leiomyosarcoma}

Leiomyosarcomas are rare typically large lesions $(>10 \mathrm{~cm})$ presenting as solitary masses with areas of haemorrhage and necrosis that may bulge into and distort the uterine cavity, but the epicentre is in the myometrium (Kurman et al. 2014).

Pelvic ultrasound is commonly the first-line study to evaluate women for potential uterine pathology. Some findings such as heterogeneous echo texture, central necrosis and colour Doppler findings of irregular vessel distribution might suggest the diagnosis of leiomyosarcoma; however sonographic evaluation is highly unspecific and hard to differentiate from leiomyomas (the most common myometrial tumour) (Amant et al. 2009).

Leiomyosarcomas commonly cause massive uterine enlargement, demonstrating heterogeneous low to intermediate signal intensity and scattered hyperintense foci on T1-weighted images (WI), whereas on T2-WI they are irregular and ill-defined lesions with intermediate to high signal intensity with cystic areas denoting necrosis (present in $>50 \%$ of cases) (Santos and Cunha 2015). Detection of scattered foci of haemorrhages or necrosis may suggest the diagnosis; however signal intensity alone is not a reliable indicator of malignancy (Amant et al. 2009) (Fig. 1).

As stated before, leiomyosarcomas and leiomyomas are independent entities with differing cytogenetic abnormalities that can however coexist (Bodner et al. 2003; Hodge and Morton 2007; 

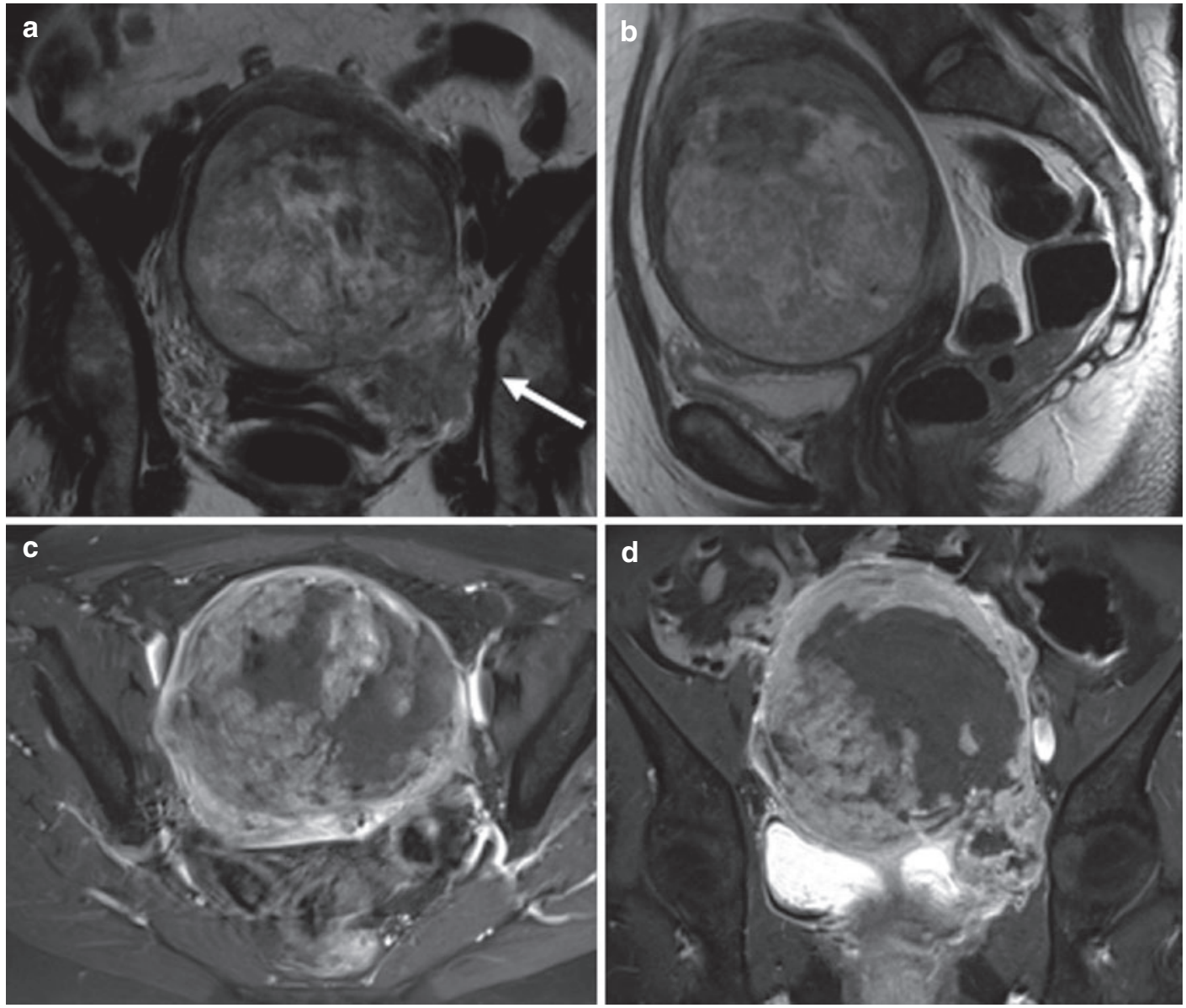

Fig. 1 Leiomyosarcoma in a 56-year-old woman. Coronal (a) and sagittal (b) T2-WI show marked uterine enlargement due to a heterogeneous myometrial tumour that invades the pelvic wall on the left (arrow), with

intense enhancement in solid areas showed here in axial (c) and coronal (d) gadolinium-enhanced T1-WI with fat suppression and extensive areas of tumoural necrosis

McCluggage 2002a). Benign leiomyomas develop primarily in women of reproductive age and typically stabilize or diminish in size following menopause. In contrast, increasing age is a significant risk factor for uterine sarcomas, and new or a rapidly growing uterine mass warrants further evaluation in menopausal women.

There is however a considerable overlap in the MR appearance, signal intensity and enhancement characteristics of leiomyosarcoma and leiomyomas with atypical imaging features (Schwartz et al. 1998; Cornfield et al. 2010). As so the diagnosis of uterine sarcomas can be incidental in histopathology analysis following hysterectomy (Sagae et al. 2004). It should also

be highlighted that with the development of laparoscopic surgery, morcellation of presumed leiomyomas has resulted in suboptimal treatment in patients with previously undiagnosed uterine sarcomas and reinforcing the need of better preoperative tumour characterization (Sutton 2013).

It has been suggested that an irregular contour, high signal on T2-WI and hyperintense areas on T1-WI could favour leiomyosarcoma against leiomyoma, but the specificity of this finding has not been established (Cornfield et al. 2010; Murase et al. 1999; Tanaka et al. 2004). The absence of calcifications is also a consistent finding in leiomyosarcomas (Schwartz et al. 1998). 

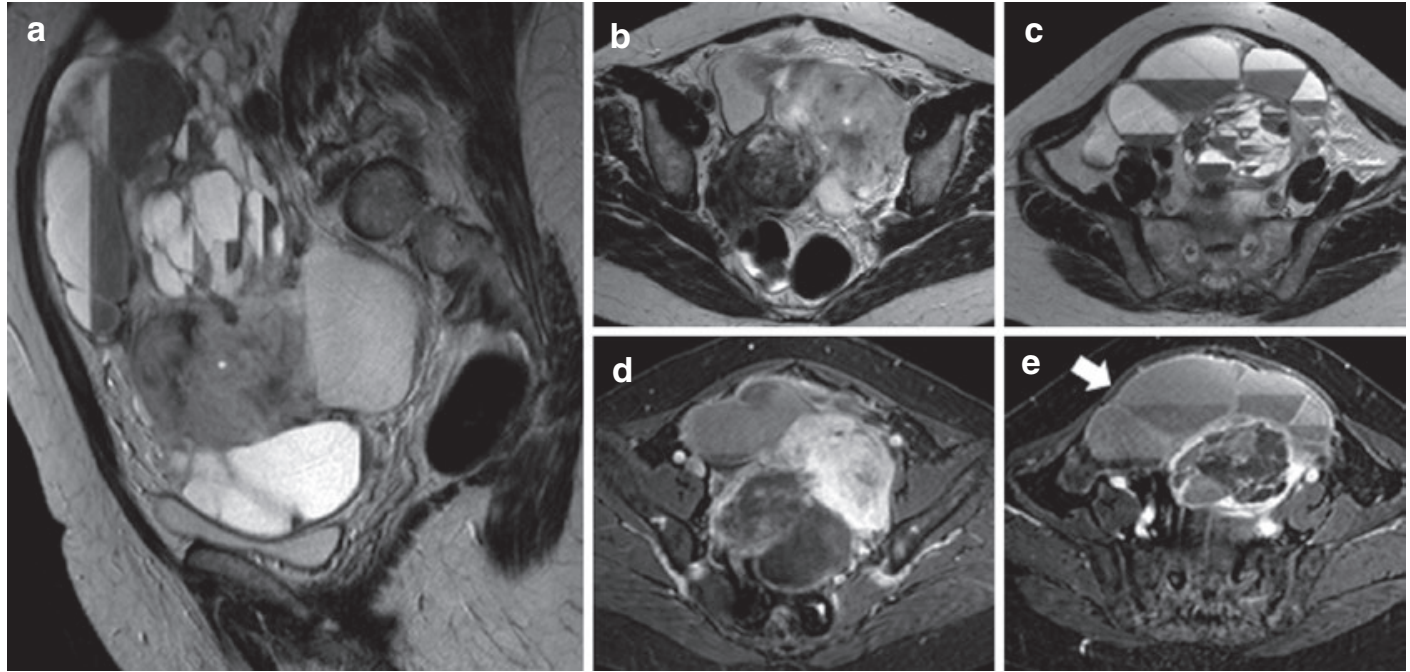

Fig. 2 Myxoid leiomyosarcoma in a 60-year-old, appearing to grow from a solid anterior myometrial nodule (asterisk on sagittal - a and axial T2-WI - b) and communicating with an exuberant multiloculated cystic component, with multiple fluid levels (c). On axial T1-WI with fat saturation and contrast administration ( $\mathbf{d}$ and $\mathbf{e}$ ), some levels have high signal (arrow) related to haemorrhagic content
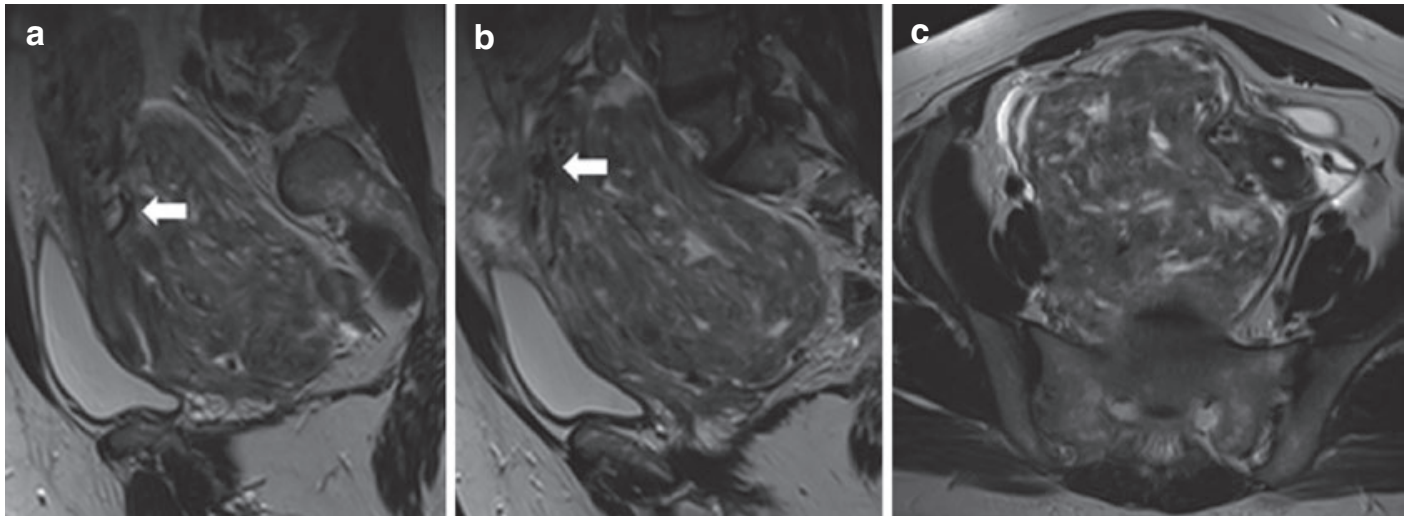

Fig. 3 Epithelioid leiomyosarcoma in a 43-year-old woman. This subserosal tumour arises from the right posterior uterine wall. On sagittal T2-WI (a and b) the flow

The myxoid and epithelioid variants can have a more diverse appearance, and the establishment of the diagnosis is particularly difficult in these cases (Figs. 2 and 3).

Leiomyosarcomas demonstrate early haematogenous spread to the lungs and liver (Sala et al. 2013). Involvement of pelvic lymph nodes is uncommon and usually associated with advanced intra-abdominal disease and decreased survival (Leitao et al. 2003; Kapp et al. 2008) (Fig. 4). voids of the connecting vessels are easily seen (arrows). On axial T2-WI (c) this lesion is seen on the right of the uterine cervix. The endometrial cavity is indistinct

The major advantage of diffusion-weighted imaging (DWI) sequences is the delineation of malignant lesions DWI seems to be a potentially useful tool in soft tissue characterization of large uterine lesions: the high nuclear-to-cytoplasm ratio in tumour cells limits intracellular motion of water molecules in uterine sarcomas and these lesions are depicted as hyperintense lesions on DWI and low signal on the corresponding apparent diffusion coefficient (ADC) maps. 

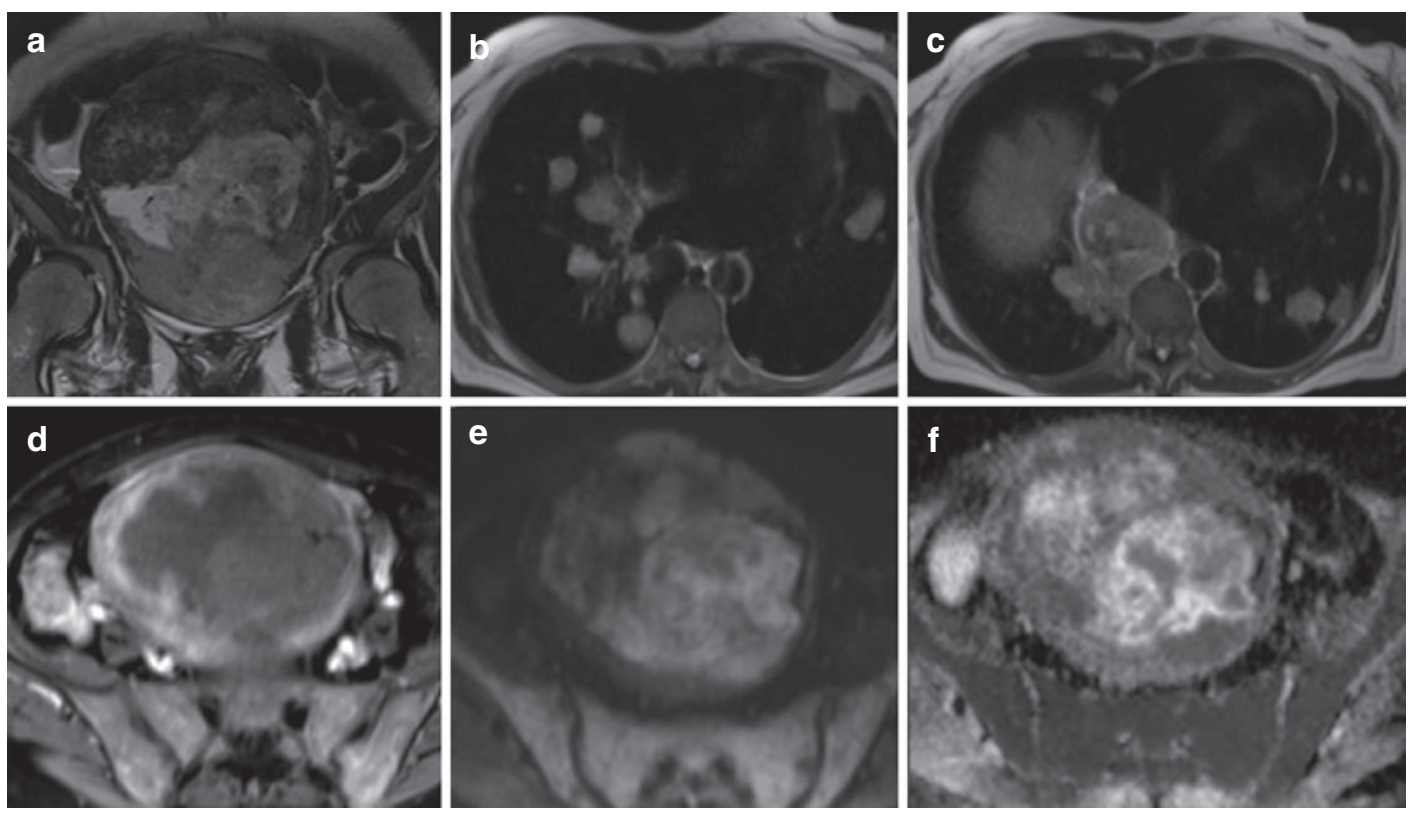

Fig. 4 Leiomyosarcoma in a 55-year-old woman. Coronal $\mathrm{T} 2$-WI of the pelvis (a) shows marked uterine enlargement due to a heterogeneous myometrial tumour with areas of high signal. Axial T2-WI, at the level of the pulmonary bases (b and $\mathbf{c}$ ), reveals pulmonary metastases and posterior mediastinum enlarged lymph nodes (c). In axial

Leiomyosarcomas display restriction in DWI studies.

DWI proved to reduce misdiagnoses of uterine sarcoma as benign leiomyoma with high accuracy (Thomassin-Naggara et al. 2013). DWI combined with T2-WI is even better than DWI alone in the differentiation of uterine sarcomas from benign leiomyomas (Namimoto et al. 2009). It is however important to recall, as a potential pitfall, that non-malignant lesions with high cellular density, such as high celular leiomyomas, may have restricted diffusion.

Positron emission tomography (PET) combined with $\mathrm{CT}$ adds metabolic information to morphologic data; However the main role of PET-CT is confined to post-therapy surveillance in order to detect distant metastasis or local recurrence, it appears to exist a significant variability in fluorodeoxyglucose (FDG) mean standard uptake values (SUVs) between benign and malignant neoplasms (Tirumani et al. 2013; Kao et al. 2011). gadolinium-enhanced T1-WI with fat suppression (d), the tumour demonstrates early intense peripheral enhancement with irregular central zones of low signal intensity denoting extensive necrosis. Endometrial cavity is difficult to depict. DWI-MR (e) and ADC map (f) show restricted diffusion in the solid areas of the lesion

\subsection{Endometrial Stromal Sarcomas}

The ultrasonographic evaluation of these lesions is highly unspecific. They appear as heterogeneous hypoechoic endometrial masses with irregular endometrial-myometrial interface, sometimes with fingerlike invasive myometrial projections and low-resistance intralesional arteries on Doppler (Amant et al. 2009; Gandolfo et al. 2000).

T2-WI are the most helpful MR sequences in detecting the endometrial nature of the tumours and its relationships with surrounding myometrium (Gandolfo et al. 2000).

Another feature that may suggest endometrial stromal sarcoma, observed in about one third of the cases, is the continuous extension of the lesion into adjacent structures most commonly the ovary, but also the fallopian tubes, the surrounding ligaments and vessels (Koyama et al. 1999). Digitiform tumour extension is best delineated on DWI (Tamai et al. 2008), differing from 

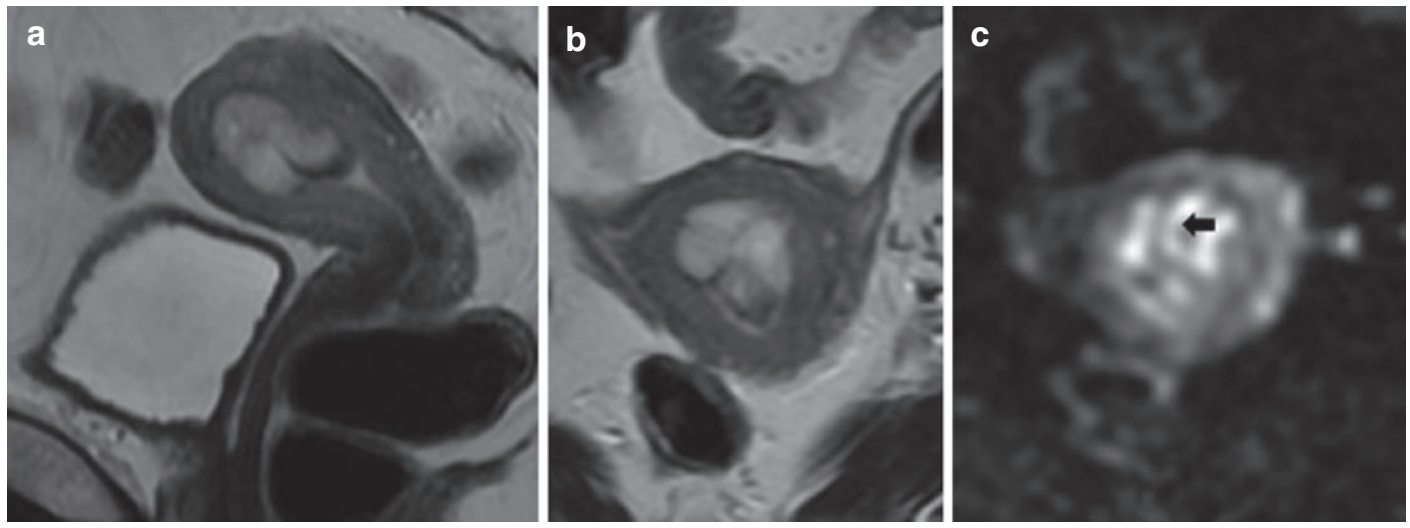

Fig. 5 Low-grade endometrial stromal sarcoma in a 62 -year-old woman with postmenopausal bleeding. Sagittal (a) and axial (b) T2-WI of the pelvis demonstrate a high signal endometrial polypoid lesion, with ill-defined endometrial/myometrial border and without evidence of a

intravenous leiomyomatosis due to tumoural high signal (Riopel et al. 2005).

Lymph node metastases occur in up to $30 \%$ of patients, and besides pelvic chain, spread to paraaortic nodes has also been reported (Fekete and Vellios 1984).

\subsubsection{Low-Grade Endometrial Stromal Sarcoma}

These tumours occur in a younger age group compared to high-grade lesions, usually involve the endometrium forming a polyp of homogeneous high signal intensity on T2-WI, which may be infarcted or haemorrhagic (Fig. 5).

There is myometrial invasion and an infiltrative growth pattern that may form deceptively well-demarcated nodules in the myometrium or may cause myometrial diffuse thickening without evidence of a well-defined tumour (Tamai et al. 2008). In some cases this diffuse thickening can be been misinterpreted as adenomyosis and, if more localized, can present as a myometrial mass mimicking a degenerated cystic leiomyoma, however without the typical well-defined border. In the presence of a large myometrial lesion, it is extremely important to evaluate the adjacent endometrial thickness, endometrial-myometrial border and lesion margin (Koyama et al. 1999).

On MRI, these lesions have heterogeneous signal intensity on both T1- and T2-WI (Gandolfo well-defined myometrial mass. DWI-MRI (c) allowed better identification of the lesion and revealed bands of low signal intensity (arrow) representing preserved bundles of the myometrium. Histopathology revealed internal myometrial invasion

et al. 2000; Koyama et al. 1999), and the most important imaging feature is the presence of bands of low signal intensity on T2-WI invading the myometrium, representing preserved muscular bundles.

Tumour enhancement is commonly heterogeneous and iso- or hyperintense when compared with normal myometrium (in contrast to endometrial carcinoma, which enhances less than normal myometrium) (Sala et al. 2013). Sometimes signal voids can be observed reflecting the hypervascular nature of these tumours (Koyama et al. 1999).

Although low-grade endometrial stromal sarcomas have an indolent clinical course there is a significant tendency for recurrence (Fig. 6).

\subsubsection{High-Grade Endometrial Stromal Sarcoma}

Recently a subset of endometrial stromal sarcomas has been discovered with a unique gene rearrangement, distinct morphologic features and an intermediate prognosis between low-grade and undifferentiated stromal sarcomas, supporting the need to consider these tumours a separated category (Lee et al. 2012).

On MRI, the T2-WI hypointense preserved bundles of myometrial fibres are much thinner, scattered and harder to depict compared to lowgrade lesions, due to a more aggressive growth (Koyama et al. 1999) (Fig. 7). 

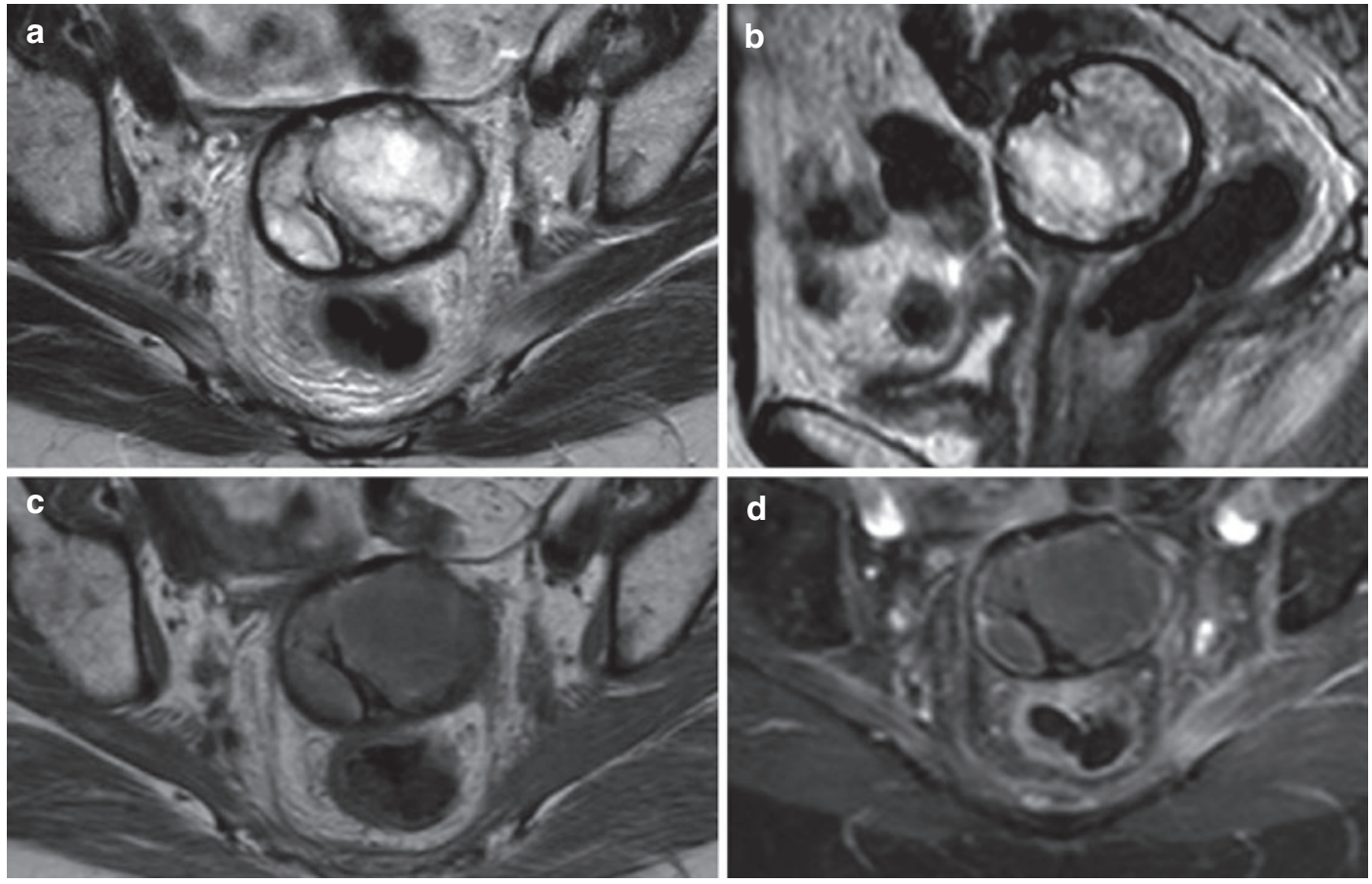

Fig. 6 Low-grade endometrial stromal sarcoma recurrence in a 70-year-old woman 2 years after surgery. Axial (a) and sagittal (b) T2-WI images demonstrate a large heterogeneous cystic and solid mass in the vaginal cuff. On

axial T1-WI (c) the lesion in hypointense, with faint enhancement after contrast on T1-WI fat supressed acquisition (d).
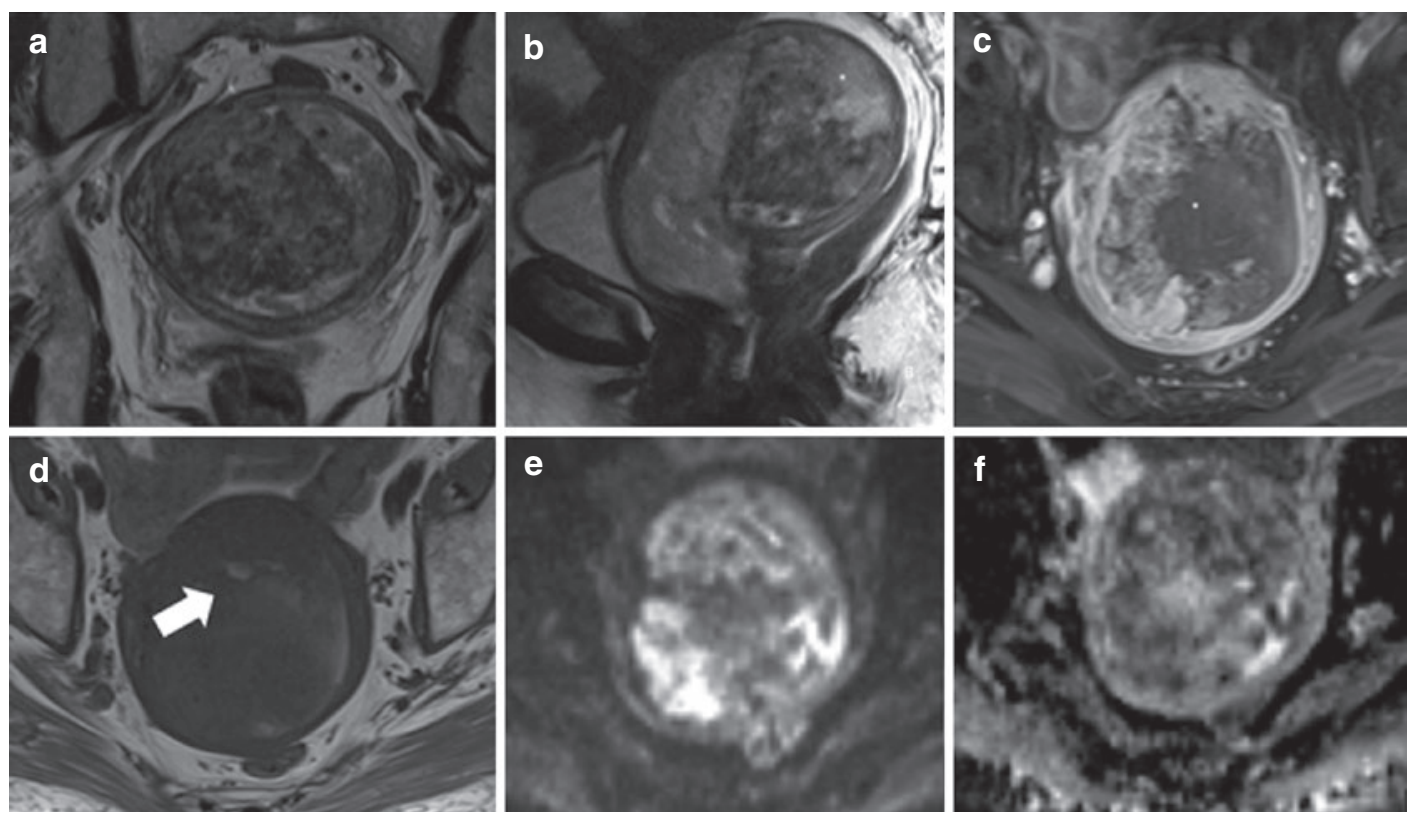

Fig. 7 High-grade endometrial stromal sarcoma in a 75-year-old woman. Coronal (a) and sagittal T2-WI (b) revealed a heterogeneous mass with necrotic areas (aster$i s k$ ) extending to the cervix, with heterogeneous enhancement after gadolinium administration (c), high-signal foci on T1-WI (d) (arrow) denoting haemorrhage, and marked restriction on DWI (e) and ADC map (f). The gross examination of the specimen revealed a heterogeneous endometrial mass invading the myometrium and reaching the cervix denoting aggressive growth 

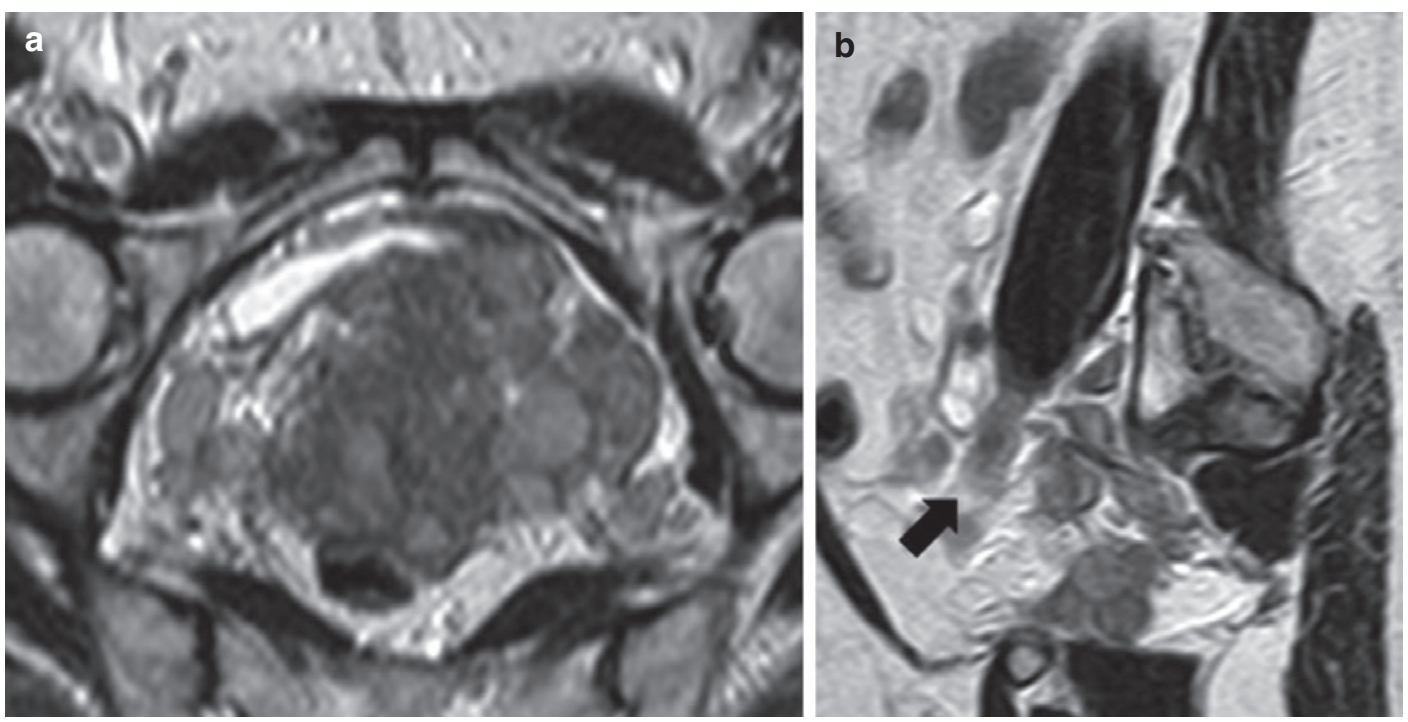

Fig. 8 High-grade endometrial stromal sarcoma in a 64-year-old woman. Axial (a) and sagittal (b) T2-WI show a heterogeneous multinodular tumour with exten-

The tumoural extension to adjacent organs, supporting structures and vascular elements is also characteristic (Fig. 8).

\subsubsection{Undifferentiated Uterine Sarcoma}

This is a tumour arising in the endometrium or the myometrium, lacking any resemblance to proliferative-phase endometrial stroma.

There are few studies describing the characteristic appearance of undifferentiated uterine sarcoma, suggesting that they appear similarly to high-grade endometrial stromal sarcomas and as so presenting as polypoid masses that frequently fill the endometrial cavity, with irregular margins, multiple marginal tumour nodules, extensive areas of haemorrhage, necrosis and myometrial invasion (Tirumani et al. 2013) (Fig. 9).

These tumours are typically characterized by a haphazard arrangement of variably sized blood vessels, as so manifesting as enhancing masses that can have marked vascular and lymphatic invasion (Amant et al. 2009; Santos and Cunha 2015) (Fig. 10).

Also metastatic extrauterine disease is more frequent than in the previously described types of endometrial stromal sarcoma (Tanner et al. 2012). sive myometrial invasion and high-signal tumoural extension along pelvic vessels (black arrow)

\subsection{Adenosarcoma}

The majority of adenosarcomas are present in the endometrium, but they may also arise from the myometrium, cervix or extrauterine Müllerian tissues (Huang et al. 2014).

Sonographic features of adenosarcoma may include expansion of endometrial cavity associated with a thickened heterogeneous and cystic complex mass. These features can mimic gestational trophoblastic disease (Santos and Cunha 2015; Chourmouzi et al. 2003).

These neoplasms present as polypoid solid, well-circumscribed multiseptated cystic mass with heterogeneous solid components. They usually arise from the fundus, sometimes protrude through the cervical os, and typically cause marked enlargement of the uterus with a thin myometrium (Amant et al. 2009; Santos and Cunha 2015; Chourmouzi et al. 2003) (Fig. 11). On MR the cysts have hight signal on T2-WI, representing glandular epithelial components or necrosis, solid component shows T2-WI hypointensity, resulting in a latticework appearance due to the intervening septa that enhance after contrast (Chourmouzi et al. 2003; Szklaruk et al. 2003) (Fig. 12). 

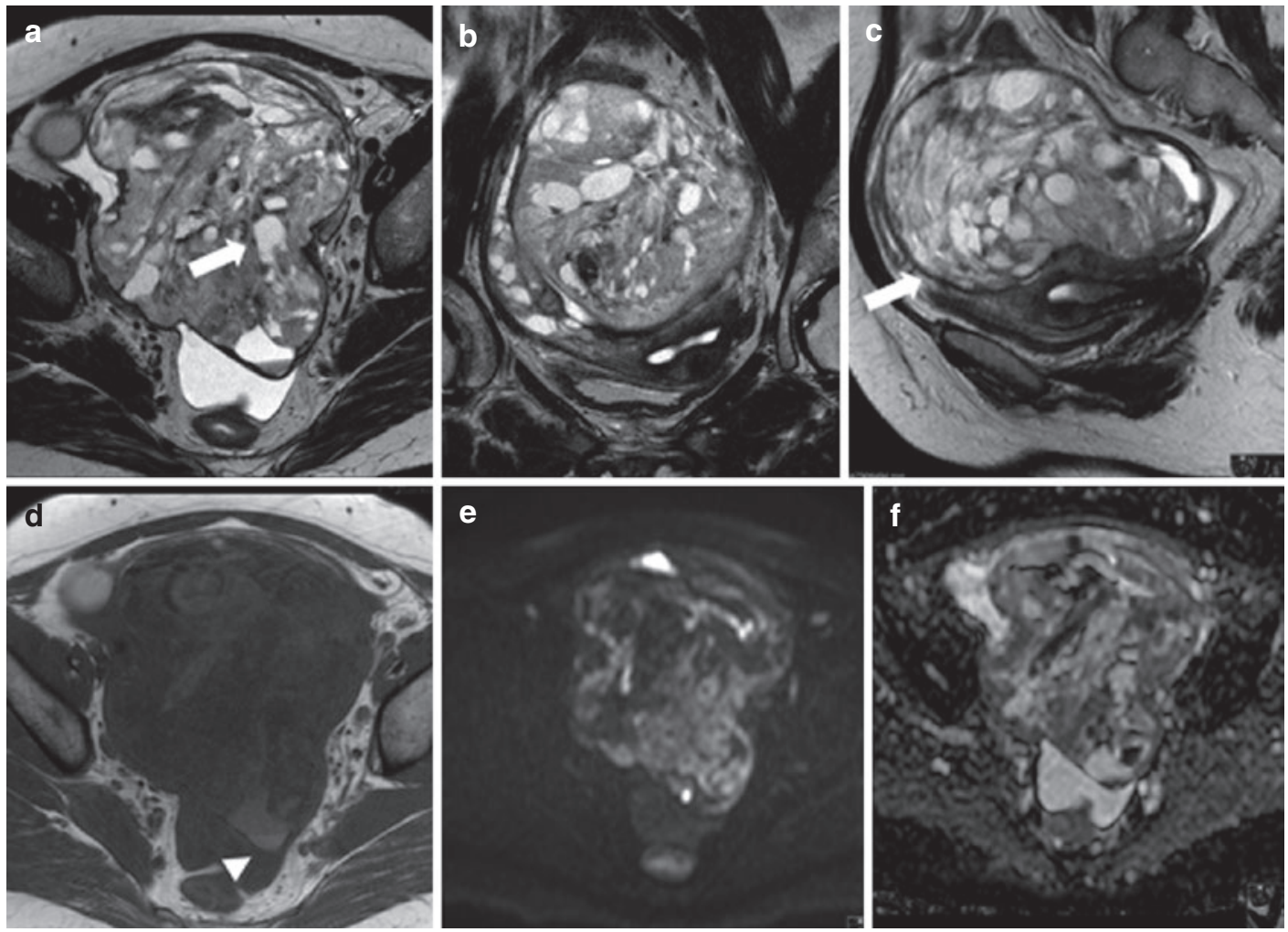

Fig. 9 Undifferentiated uterine sarcoma in a 56-year-old woman. Axial (a), coronal (b) and sagittal (c) T2-WI show marked uterine enlargement due to a large subserosal heterogeneous tumour, with lobulated contours, showing

extensive cystic and necrotic areas (arrows). On T1-weighted image (d) haemorrhage can be appreciated (arrow-head). The tumour shows marked restriction on DWI (e) and on the ADC map (f)
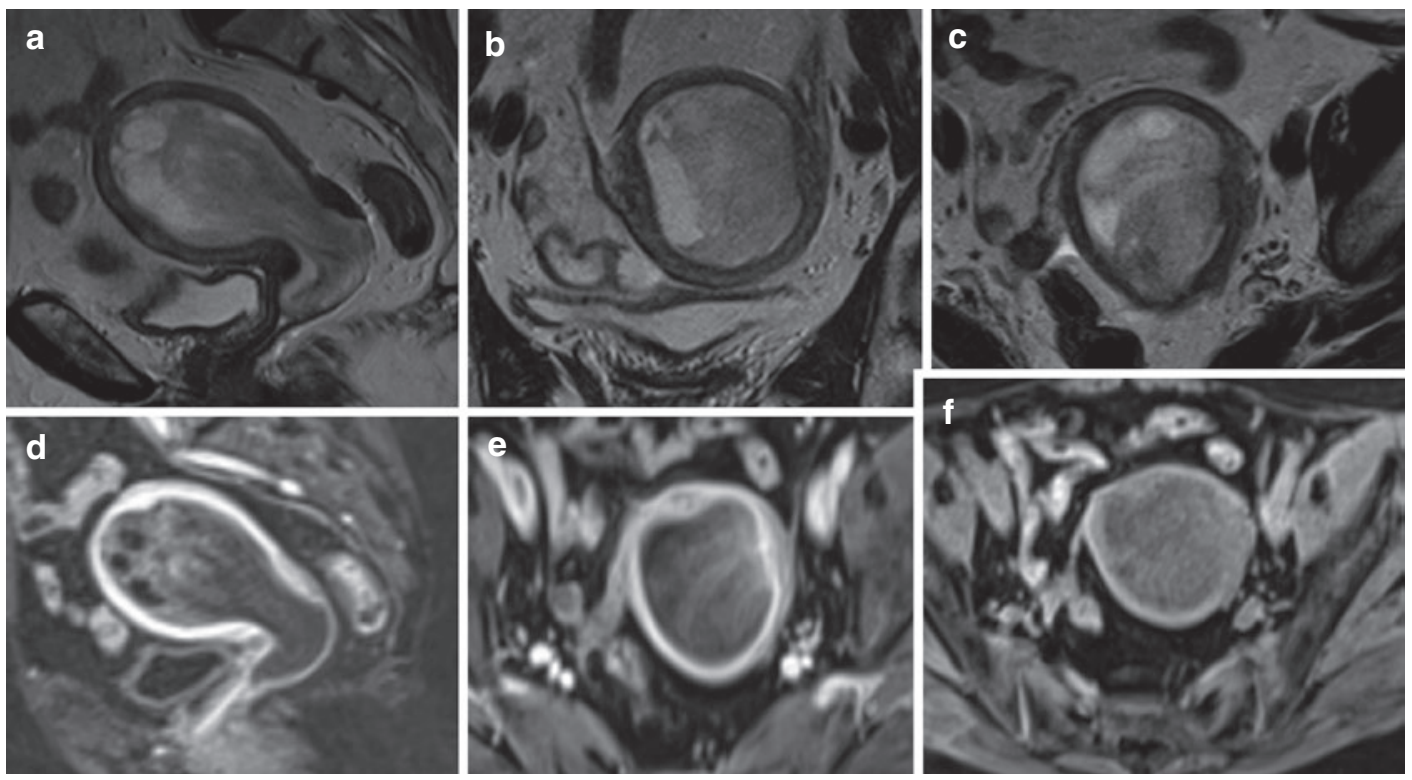

Fig. 10 Undifferentiated uterine sarcoma in a 72-yearold woman. Sagittal (a), coronal (b) and axial and (c) T2-WI show a polypoid mass with heterogeneous high signal intensity within the endometrial cavity, protruding through the cervical os into the vagina. On T1-WI FS after gadolinium administration, in the same three planes $(\mathbf{d}-\mathbf{f})$ the lesion enhances heterogeneously compared to the peripheral myometrium 


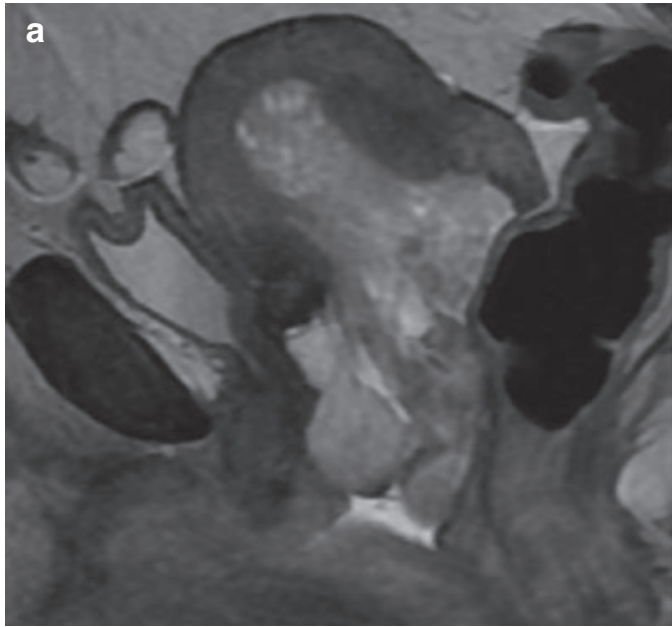

Fig. 11 Adenosarcoma in a 48-year-old woman presenting with pelvic mass and vaginal discharge. Sagittal (a) and axial (b) T2-WI demonstrate a large, heterogeneous,

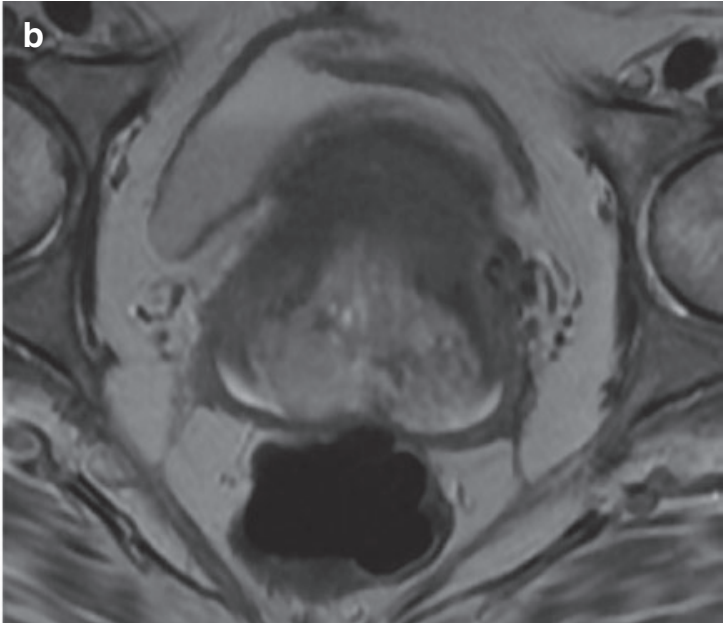

high-signal intensity mass centred in the endometrial cavity, protruding trough the cervical canal and prolapsing
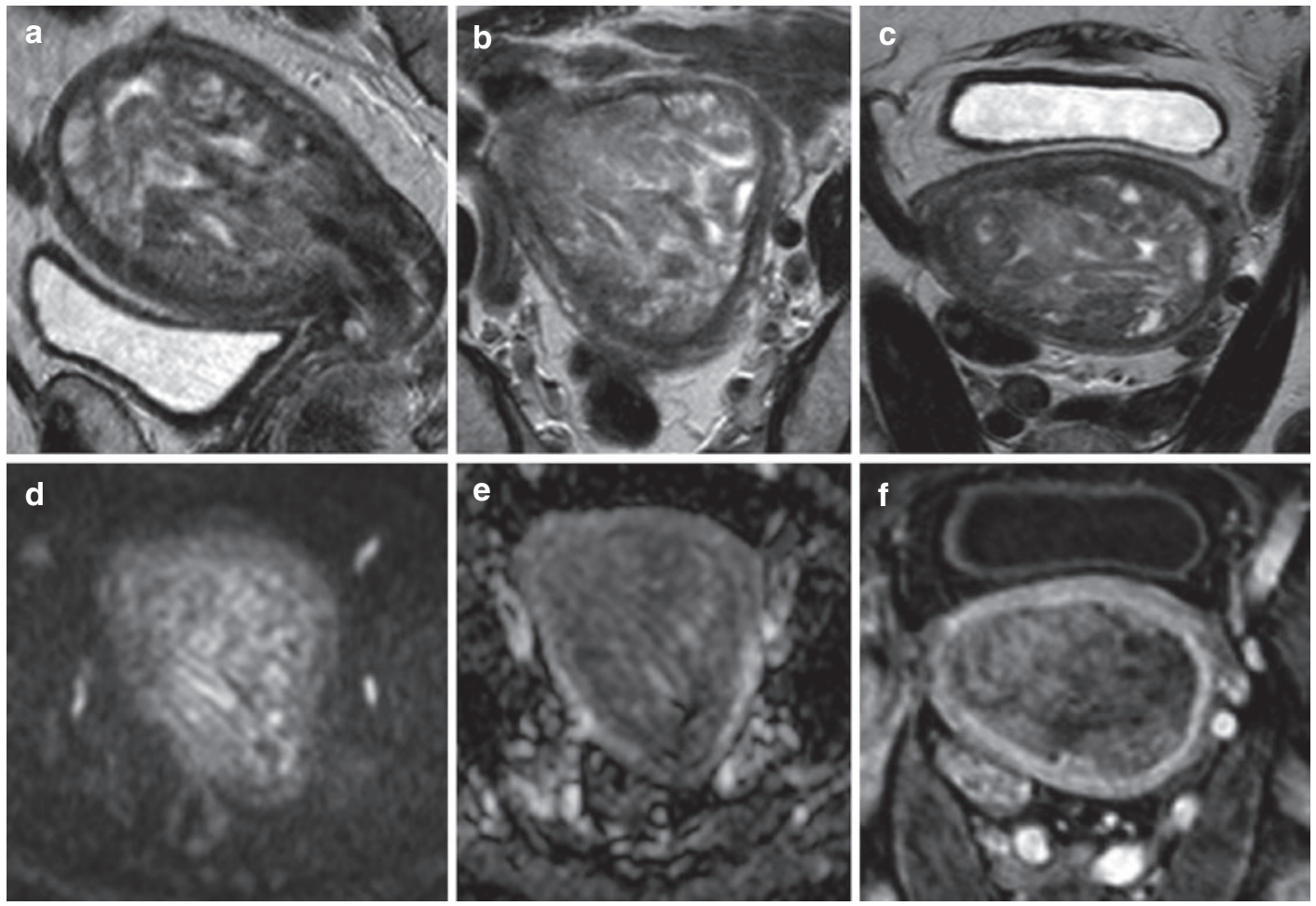

Fig. 12 Adenosarcoma in a 77-year-old woman presenting with post-menopausal bleeding. Sagittal (a), axial (b) and axial of the uterine body (c) T2-WI of the pelvis demonstrate a huge endometrial mass with cystic spaces and heterogeneous solid components. The uterine contour is preserved. Axial DWI-MR (d) reveals high signal intensity and low signal on the ADC map (e). T1-WI fat suppressed after administration of contrast (f) shows early enhancement of the septa and solid areas. No myometrial invasion was present on pathology 
The cases of adenosarcoma with sarcomatous overgrowth are described in the literature as large pelvic masses with heterogeneous signal intensity, cystic areas and transperitoneal extension with contiguous intravascular growth (Kim et al. 2011).

\section{Prognosis and Treatment}

Overall 5-year survival for uterine sarcomas ranges between around $18-55 \%$, considerably lower than for endometrial carcinomas (Koivisto-Korander et al. 2008). Prognostic factors include age, clinical stage, tumour size, tumour circumscription, mitotic index and lymphovascular invasion (D'Angelo and Prat 2010; Abeler et al. 2009).

Leiomyosarcomas and undifferentiated uterine sarcomas are highly aggressive neoplasms with a marked propensity for extrauterine spread and systemic metastasis; and nodal metastasis is not so common (McCluggage et al. 2014; Kapp et al. 2008).

In undifferentiated uterine sarcomas, a significant proportion of patients $(>60 \%)$ has advancedstage disease at the time of diagnosis, with extrauterine spread of tumour to the upper abdomen, pelvic lymph nodes or even more distant sites such as the lungs (Leath et al. 2007).

On the other hand, low-grade endometrial stromal sarcomas have an indolent clinical course with good long-term survival, despite the tendency for late recurrence. Stage is the most important prognostic factor, with FIGO stage I and II tumours having an excellent 5-year survival rate.

Adenosarcomas are mixed tumours of low malignant potential containing a benign epithelial and a malignant stromal component, usually of low grade. They usually have a favourable prognosis with a 5-year survival rate above $80 \%$ unless associated with sarcomatous overgrowth or deep myometrial invasion (McCluggage et al. 2014; Abeler et al. 2009).

The current standard of care for uterine sarcomas remains surgery: total abdominal hysterectomy with bilateral salpingo-oophorectomy.
In younger patients preservation of the ovaries can be considered in early-stage disease.

For patients with evidence of extrauterine disease, surgical staging and cytoreduction are performed only if intra-abdominal metastases are resectable and there is no extra-abdominal disease. If patients are not surgical candidates, medical treatment should be offered. Whether systematic lymphadenectomy is needed is still controversial (Prat and Mbatani 2015).

Adjuvant chemotheraphy is decided in a caseto-case base and not consensually indicated. Radiotherapy is also not routinely performed as it does not appear to provide a benefit in survival, but it may reduce local recurrence in high-risk women (Seddon and Davda 2011).

Recurrence can be treated either by surgical resection or by systemic chemotherapy, with hormonotherapy in particular cases, when hormonal receptors are present (Amant et al. 2009).

\section{References}

Abeler VM, Royne O, Thoresen S et al (2009) Uterine sarcomas in Norway. A histopathological and prognostic survey of a total population from 1970 to 2000 including 419 patients. Histopathology 54:355-364

Ali RH, Rouzbahman M (2015) Endometrial stromal tumours revisited: an update based on the 2014 WHO classification. J Clin Pathol 68(5):325-332

Amant F, Coosemans A, Debiec-Rychter M, Timmerman D, Vergote I (2009) Clinical management of uterine sarcomas. Lancet Oncol 10:1188-1198

Blom R, Guerrieri C (1999) Adenosarcoma of the uterus: a clinicopathologic, DNA flow cytometric, p53 and mdm-2 analysis of 11 cases. Int J Gynecol Cancer 9(1):37-43

Bodner K, Bodner-Adler B, Kimberger O et al (2003) Estrogen and progesterone receptor expression in patients with uterine leiomyosarcoma and correlation with different clinicopathological parameters. Anticancer Res 23:729

Chan JK, Kawar NM, Shin JY et al (2008) Endometrial stromal sarcoma: a population-based analysis. $\mathrm{Br}$ J Cancer 99(8):1210-1215

Chourmouzi D, Boulogianni G, Zarampoukas T, Drevelengas A (2003) Sonography and MRI of tamoxifen-associated Mullerian adenosarcoma of the uterus. AJR Am J Roentgenol 181:1673-1675

Conklin CM, Longacre TA (2014) Endometrial stromal tumours: the new WHO classification. Adv Anat Pathol 21(6):383-393 
Cornfield D, Israel G, Martel M et al (2010) MRI appearance of mesenchymal tumors of the uterus. Eur J Radiol 74:241-249

D’Angelo E, Prat J (2010) Uterine sarcomas: a review. Gynecol Oncol 116:131-139

Fekete PS, Vellios F (1984) The clinical and histologic spectrum of endometrial stromal neoplasms: a report of 41 cases. Int J Gynecol Pathol 3:198-212

Felix AS, Cook LS, Gaudet MM et al (2013) The etiology of uterine sarcomas: a pooled analysis of the epidemiology of endometrial cancer consortium. Br J Cancer 108:727-734

Gandolfo N, Gandolfo NG, Serafini G, Martinoli C (2000) Endometrial stromal sarcoma of the uterus: MR and US findings. Eur Radiol 10:776-779

Hodge JC, Morton CC (2007) Genetic heterogeneity among uterine leiomyomata: insights into malignant progression. Hum Mol Genet 16(1):R7

Huang PS, Chang WC, Huang SC (2014) Müllerian adenosarcoma: a review of cases and literature. Eur J Gynaecol Oncol 35(6):617-620

Kao YH, Saad U, Tan AE, Magsombol BM, Padhy AK (2011) Fluorine-18-fluorodeoxyglucose PET/CT for the evaluation of suspected recurrent uterine leiomyosarcomas. Acta Radiol 52:463-466

Kapp DS, Shin JY, Chan JK (2008) Prognostic factors and survival in 1396 patients with uterine leiomyosarcomas: emphasis on impact of lymphadenectomy and oophorectomy. Cancer 112:820-830

Kim SA, Jung JS, Ju SJ, Kim YT, Kim KR (2011) Mullerian adenosarcoma with sarcomatous overgrowth in the pelvic cavity extending into the inferior vena cava and the right atrium. Pathol Int 61:445-448

Koivisto-Korander R, Butzow R, Koivisto AM, Leminen A (2008) Clinical outcome and prognostic factors in 100 cases of uterine sarcoma: experience in Helsinki University Central Hospital 1990-2001. Gynecol Oncol 111:74-81

Koyama T, Togashi K, Konishi I et al (1999) MR imaging of endometrial stromal sarcoma: correlation with pathologic findings. AJR Am J Roentgenol 173:767-772

Kurman RJ, Carcangiu ML, Herrington S, Young RH (eds) (2014) WHO classification of tumours of the female reproductive organs, $4 \mathrm{edn}$. IARC, Lyon

Leath CA III, Huh WK, Hyde J Jr et al (2007) A multiinstitutional review of outcomes of endometrial stromal sarcoma. Gynecol Oncol 105:630-634

Lee C-H, Mariño-Enriquez A, Ou W et al (2012) The clinicopathologic features of YWHAE-FAM22 endometrial stromal sarcomas: a histologically high-grade and clinically aggressive tumor. Am J Surg Pathol 36:641-653

Leitao MM, Sonoda Y, Brennan MF, Barakat RR, Chi DS (2003) Incidence of lymph node and ovarian metastases in leiomyosarcoma of the uterus. Gynecol Oncol 91(1):209-212

Major FJ, Blessing JA, Silverberg SG et al (1993) Prognostic factors in early stage uterine sarcoma. A Gynecologic Oncology Group study. Cancer 71(Suppl 4):1702-1709
McCluggage WG (2002a) Malignant biphasic uterine tumours: carcinosarcomas or metaplastic carcinomas? J Clin Pathol 55:321-325

McCluggage WG (2002b) Uterine carcinosarcomas (malignant mixed Mullerian tumors) are metaplastic carcinomas. Int J Gynecol Cancer 12:687-690

McCluggage WG, Fisher C, Hirschowitz L; On behalf of the Working Group for Cancer Services of The Royal College of Pathologists (2014) Standards and datasets for reporting cancers. Dataset for histological reporting of uterine sarcomas. London: The Royal College of Pathologists

Murase E, Siegelman ES, Outwater EK, Perez-Jaffe LA, Tureck RW (1999) Uterine leiomyomas: histopathologic features, MR imaging findings, differential diagnosis, and treatment. Radiographics 19(5):1179-1197

Namimoto T, Yamashita Y, Awai K et al (2009) Combined use of T2-weighted and diffusion-weighted 3-T MR imaging for differentiating uterine sarcomas from benign leiomyomas. Eur Radiol 19:2756-2764

Prat J (2009a) Corrigendum to 'FIGO staging for uterine sarcomas' [Int J Gynaecol Obstet 2009; 104:179]. Int J Gynaecol Obstet 106:277

Prat J (2009b) FIGO staging for uterine sarcomas. Int J Gynaecol Obstet 104:177-178

Prat J, Mbatani N (2015) Uterine sarcomas. Int J Gynaecol Obstet 131(Suppl 2):S105-S110

Riopel J, Plante M, Renaud M-C et al (2005) Lymph node metastases in low-grade endometrial stromal sarcoma. Gynecol Oncol 96:402-406

Sagae S, Yamashita K, Ishioka S et al (2004) Preoperative diagnosis and treatment results in 106 patients with uterine sarcoma in Hokkaido, Japan. Oncology 67(1):33-39

Sala E, Rockall AG, Freeman SJ, Mitchell DG, Reinhold C (2013) The added role of MR imaging in treatment stratification of patients with gynecologic malignancies: what the radiologist needs to know. Radiology 266:717-740

Santos P, Cunha TM (2015) Uterine sarcomas: clinical presentation and MRI features. Diagn Interv Radiol 21(1):4-9

Schwartz LB, Zawin M, Carcangui MA, Lange R, McCarthy S (1998) Does pelvic magnetic resonance imaging differentiate among the histologic subtypes of uterine leiomyomata? Fertil Steril 70:580-587

Seddon BM, Davda R (2011) Uterine sarcomas - recent progress and future challenges. Eur J Radiol 78(1): $30-40$

Sherman ME, Devesa SS (2003) Analysis of racial differences in incidence, survival, and mortality for malignant tumors of the uterine corpus. Cancer 98:176-186

Sutton G (2013) Uterine sarcomas 2013. Gynecol Oncol 130(1):3-5

Szklaruk J, Tamm EP, Choi H, Varavithya V (2003) MR imaging of common and uncommon large pelvic masses. Radiographics 23:403-424

Tamai K, Koyama T, Saga T et al (2008) The utility of diffusion-weighted MR imaging for differentiating uterine sarcomas from benign leiomyomas. Eur Radiol 18:723-730 
Tanaka YO, Nishida M, Tsunoda H, Okamoto Y, Yoshikawa H (2004) Smooth muscle tumors of uncertain malignant potential and leiomyosarcomas of the uterus: MR findings. J Magn Reson Imaging 20: 998-1007

Tanner EJ, Garg K, Leitao MM Jr, Soslow RA, Hensley ML (2012) High grade undifferentiated uterine sarcoma: surgery, treatment, and survival outcomes. Gynecol Oncol 127(1):27-31

Thomassin-Naggara I, Dechoux S, Bonneau C et al (2013) How to differentiate benign from malignant myometrial tumours using MR imaging. Eur Radiol 23:2306-2314

Tirumani SH, Ojili V, Shanbhogue AK, Fasih N, Ryan JG, Reinhold C (2013) Current concepts in the imaging of uterine sarcoma. Abdom Imaging 38(2):397-411
Toro JR, Nickerson ML, Wei MH et al (2003) Mutations in the fumarate hydratase gene cause hereditary leiomyomatosis and renal cell cancer in families in North America. Am J Hum Genet 73:95-106

Tse KY, Crawford R, Ngan HY (2011) Staging of uterine sarcomas. Best Pract Res Clin Obstet Gynaecol 25(6):733-749

Wu TI, Yen TC, Lai CH (2011) Clinical presentation and diagnosis of uterine sarcoma, including imaging. Best Pract Res Clin Obstet Gynaecol 25:681-689

Xue WC, Cheung AN (2011) Endometrial stromal sarcoma of uterus. Best Pract Res Clin Obstet Gynaecol 25(6):719-732

Yu CL, Tucker MA, Abramson DH et al (2009) Causespecific mortality in long-term survivors of retinoblastoma. J Natl Cancer Inst 101:581-591 